\title{
Mathematical Formulation on Non-Newtonian Dusty Fluid over Vertical Stretching Sheet
}

\author{
Nur Syamilah Arifin², Syazwani Mohd Zokri³, Laila Amera Aziz ${ }^{1}$, Abdul Rahman Mohd Kasim ${ }^{1 *}$ and \\ Mohd Zuki Salleh ${ }^{1}$ \\ ${ }^{1}$ Applied \& Industrial Mathematics Research Group, Faculty of Industrial Sciences \& Technology, Universiti \\ Malaysia Pahang, 26300 Gambang, Kuantan, Pahang, Malaysia \\ ${ }^{2}$ Faculty of Computer and Mathematical Sciences, UniversitiTeknologi MARA (UiTM), Johor Branch, \\ PasirGudang Campus, Masai, Johor \\ ${ }^{3}$ Faculty of Computer and Mathematical Sciences, UniversitiTeknologi MARA (UiTM), Kuala Terengganu \\ Branch, Kuala Terengganu Campus, Kuala Terengganu
}

\begin{abstract}
Two-phase flow is the interaction of two different matters, namely fluid and solid that contributes to an intriguing model in the boundary layer flow problem. It is can be precisely defined as the binary mixture of the fluid and spherical particles that involve interaction between both phases on the flow region. The fascinating class of non-Newtonian fluid, particularly Casson, Williamson and Jeffrey models are further considered for fluid phase. The mathematical formulation on the respective models are presented and discussed. The adiabatic two phases is engaged throughout the entire flow process by means of no phase changes occurred. The governing equations for the proposed models are presented herein and need to undergo the boundary layer approximation as way of tackling its difficulty into a solvable form by using the order of magnitude analysis.
\end{abstract}

Keywords: Mathematical formulation, non-Newtonian fluid, dusty fluid, vertical stretching sheet

\section{INTRODUCTION}

The field of fluid dynamics has been an object of research for the past century that relates to the fluid motion with forces. As regards to fluid, the viscosity is one of its fundamental characteristic which referring to the resistance of fluid when flowing(Nakayama, 2018). There is, therefore, the used of the Newton's law of viscosity as a remarkable way to measure the viscosity that holds for the shear stress being proportional to the velocity gradient. Air, water and electrolyte are categorized as Newtonian fluid since they portrayed such behavior. However, for those fluids that opposed the law are recognized as nonNewtonian fluid (Partal and Franco, 2010), which are commonly exhibited by blood, paint, polymer and etc. This complex fluid is classified into the different kinds of models depending on their rheological behavior that can be represented by the constitutive equation, the relation of applied stresses to deformation. Generally, the model is of the three broad categories which are time independent, time-dependent and viscoplastic fluids(Nguyen and Nguyen, 2012). Investigating non-Newtonian fluids has received consideration attentiondue toits applications in the mining industry, lubrication and biomedical flows(Khan et al., 2018). The studies of non-Newtonian fluid with various conditions have been investigated by several researchers (Kasim et al., 2012; Aurangzaib et al., 2013; Arifin et al., 2018; Kasim and Shafie, 2010).

In the case of two-phase flow, the involvement of two different states of matteris considered, which can occur either between solid, liquid and gas. The solid-liquid phase for instance, is referring to the fluid having dust particles which are important in packed beds, centrifugal separation of particles, sedimentation, environment pollution, and blood rheology (Butt et al., 2017).By drawing on the concept of two-phase flow, Saffman (1962)has been able to show the stability of dusty gas, while Marble (1963) identified four similarity parameters

*Corresponding author's e-mail: rahmanmohd@ump.edu.my 
for gas-particle flow. In view of this, the flow problems concerning fluid and dust particles have led to a numerous studies under different elements, effects, as well as dimensions(Isa et al., 2016; Sulochana et al., 2016; Arifin et al., 2017).

In this paper, Casson, Williamson and Jeffrey fluids among the time-independent fluid model are selected, which primary concern to study for fluid-particle flow with the influences of aligned magnetic field and mixed convection over a vertical stretching sheet. The fluids were also examined by different authors (Hussanan et al., 2018; Nadeem et al., 2013; Hayat et al., 2011). Before proceeding to solve the two-phase flow problems, it is necessary to formulate the basic equations that are reliable. This paper first introduces the basic equations for each flow models in the vector form. Then, the boundary layer approximation is performed by applying the order of magnitude analysis. The resulting equations embodied the above arguments are finally expressed.

\section{GOVERNING EQUATIONS}

Turning now to the computation of flow problems, the modelling is carried out by solving its governing equations which are generally consist of continuity, momentum and energy equations. In addition, they are derived according to the three fundamental physical principles, which are mass is conserved, Newton's second law and energy is conserved(Anderson Jr, 2009). These laws are also applied for dust phase. The basic equation of momentum, in particular, is different for each flow case caused by the different constitutive relation of fluid model which are revealed in Table 1. Next, the formulation for two-phase flow is carried out by independently derived the governing equations for each phase (Siddiqa et al., 2015). Three fluid models that have been mentioned so far will be examined, all of which are embedded with the dust particles. Keeping in mind that for fluid phase, they share the same continuity and energy equations, however, the momentum equation is based on their respective constitutive relation.

Table 1. The constitutive relation for non-Newtonian fluid

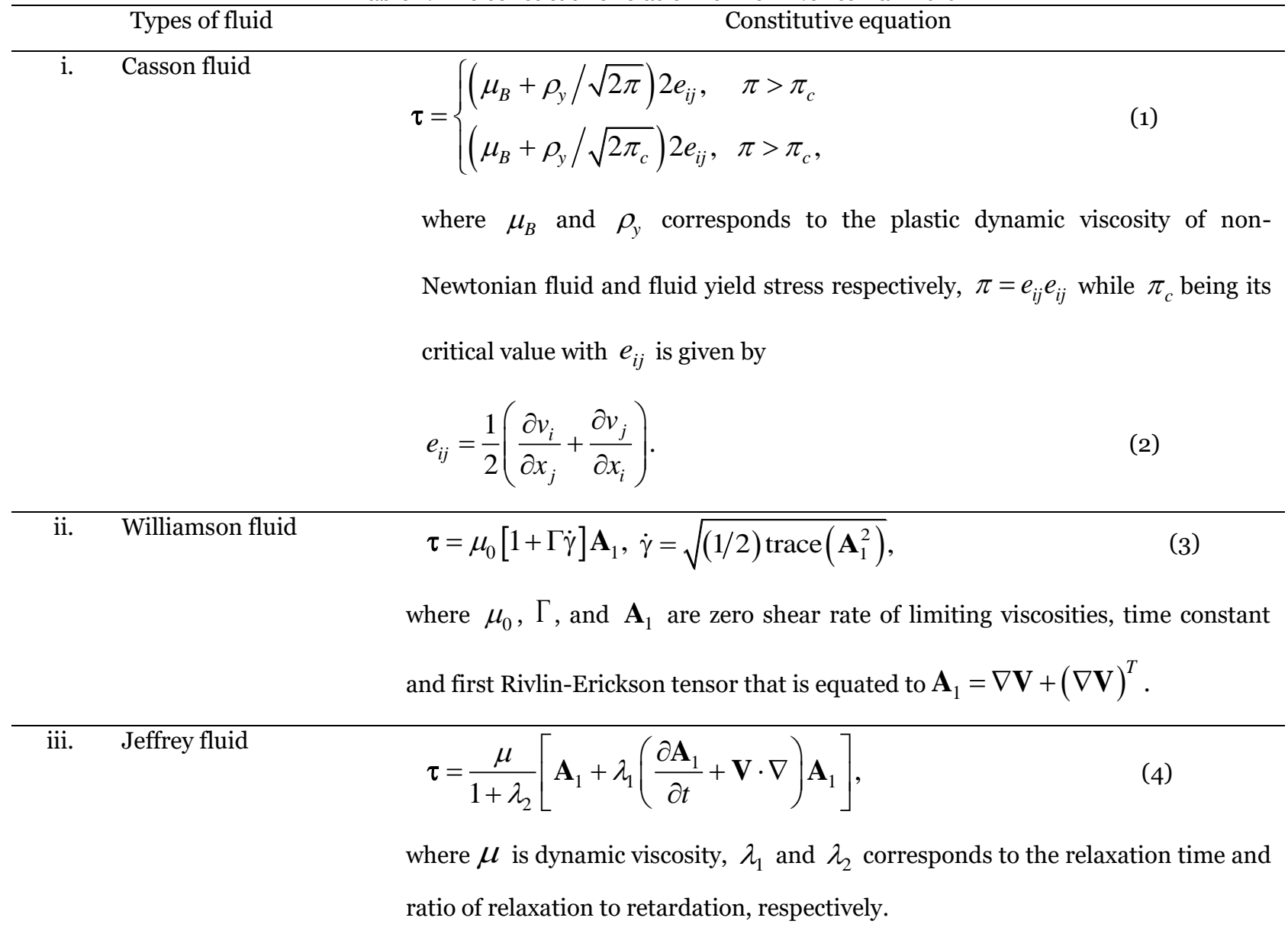


The following equations are thus expressed for fluid and dust phases as

Continuity equation:

$\nabla \cdot \mathbf{V}=0$

$\nabla \cdot \mathbf{V}_{\mathbf{p}}=0$,

Momentum equation:

$\rho\left(\frac{\partial \mathbf{V}}{\partial t}+\mathbf{V} \cdot \nabla \mathbf{V}\right)=-\nabla p+\nabla \cdot \tau+\mathbf{F}_{p}+\mathbf{F}_{b}$,

$\rho_{p}\left(\frac{\partial \mathbf{V}_{p}}{\partial t}+\mathbf{V}_{p} \cdot \nabla \mathbf{V}_{p}\right)=-\mathbf{F}_{p}$,

Energy equation:

$\rho c_{p}(\mathbf{V} \cdot \nabla) T=k \nabla^{2} T+Q_{p}$,

$\rho c_{s}(\mathbf{V} \cdot \nabla) T_{p}=-Q_{p}$,

Here, $\mathbf{V}, \tau, \mathbf{F}_{b}, T, \rho, c_{p}$ and $k$ signifies to velocity field, stress tensor, body force, temperature, density, specific heat and thermal conductivity for fluid phase. Meanwhile, $\mathbf{V}_{p}, T_{p}$, and $c_{s}$ refers to vector field, temperature and specific heat for dust phase. The total fluid-particle interaction of force and thermal per unit volumeare respectively referred to

$$
\begin{aligned}
& \mathbf{F}_{p}=6 \pi n \mu r_{p}\left(\mathbf{V}_{p}-\mathbf{V}\right), \\
& Q_{p}=4 \pi n r_{p} k\left(T_{p}-T\right) .
\end{aligned}
$$

\section{MATHEMATICAL ANALYSIS}

The boundary layer theory is originally introduced by Prandtl (1904) to reduce the complexity of governing equations into the approximate solutions in which the only significant terms are retained. In an attempt to approximate the governing equations, each term is estimated with the order of magnitude which has been reported by Schlichting (1974). By taking into account the above arguments, this approach is applied into Equations (5)-(7) to obtain the desired results in which the retaining terms are determined to be important. In the Cartesian coordinates, the steady two dimensional flow is considered in which the basic equations of fluid and dust phases are expressed in $x$ and $y$ directions with independent of time. The flow configuration of this problem is illustrated in Figure 1.

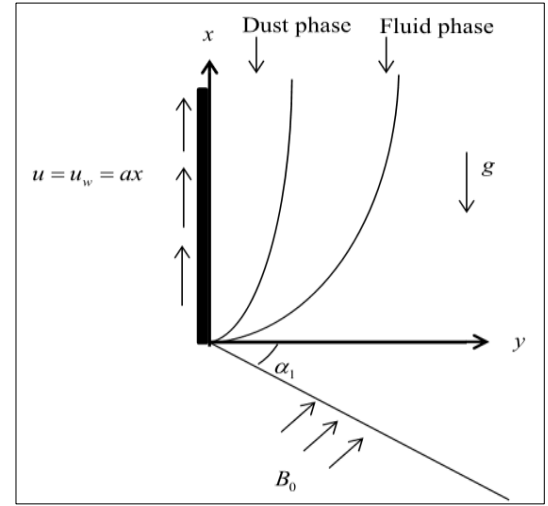

Figure 1: Flow configuration

To begin with, the terms contained in Equations (5)-(7) are estimated to be of order 1 and $\delta$.Hence, the physical quantities contained in the respective equations are given as (Nadeem et al., 2013; Schlichting, 1974; Jaluria, 1980; Kasim, 2014; Kannan, 2001)

$$
\begin{aligned}
& u \square 1, x \square 1, v \square \delta, y \square \delta, \frac{\mu}{\rho} \square \delta^{2}, \Gamma \square \delta, \\
& \sigma \sim \frac{1}{\delta^{2}}, B_{0}{ }^{2} \square \delta^{2}, \beta g\left(T-T_{\infty}\right) \square 1, T \square 1,(10) \\
& \alpha \square \delta^{2}, \lambda_{1} \square 1, \frac{v}{1+\lambda_{2}} \square \partial^{2}, \alpha \square \partial^{2}
\end{aligned}
$$$$
u_{p} \square 1, v_{p} \square \delta, \rho_{p} \square \rho, \frac{1}{\tau_{v}} \square 1, \frac{1}{\tau_{T}} \square 1, T_{p} \square l(11)
$$

\section{A. Continuity Equation}

In view of Equation (10), the continuity equation (5) is estimated and it can be seen from Table 2 that the first and second terms are of order 1 and they are considered to be retained.

Table 2. Order of magnitude for continuity equation

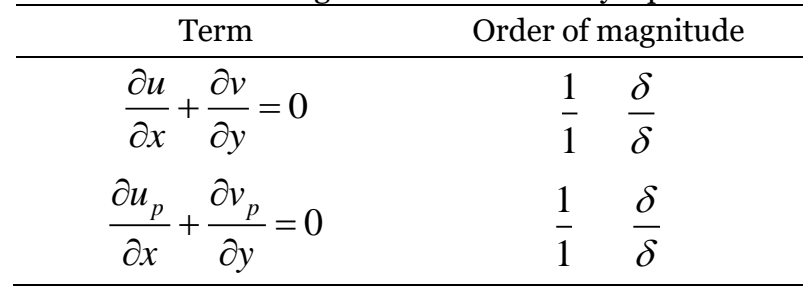




\section{B. Momentum Equation}

Substituting Equations (1)-(4) and (8) into (6) results in the momentum equation of fluid phase. Thus, the resulting equations for each fluid model are estimated by applying Equation (10) as shown in the following tables. In the case of dusty Casson fluid, term $\partial^{2} u / \partial x^{2}$ is negligible since it is of order $\delta^{2}$ which is a very small quantity as presented in Table 3. Meanwhile, the remaining terms of order 1 are retained. From Table 4 , the terms with order $\delta$ are maintained in $y$ momentum equation. This is implying to the Prandtl's theory which stated that the inertia terms and viscous terms are required to have the same order of magnitude. However, the variations of $y$ momentum in the fluid flow can be ignored stemming from all approximated terms are small quantity.

Summing up from the above elaborations, the approximation of $y$ momentum equation for the other two fluid models will not be presented. Again, Schlichting (1974) has been shown that the equation of motion in $y$ direction is completely discarded. For this reason, it is accurate enough to only estimate the momentum equation for $x$ direction and the repeating terms as contained in dusty fluid are not included in the following tables.

Table 3. Order of magnitude for $x$ momentum equation

\begin{tabular}{cc} 
Term & $1 \frac{1}{1}=1$ \\
\hline$u \frac{\partial u}{\partial x}$ & Order of magnitude \\
$v \frac{\partial u}{\partial y}$ & $\delta^{2} \frac{1}{\delta}=1$ \\
\hline$\frac{\mu_{B}}{\rho}\left(1+\frac{1}{A}\right) \frac{\partial^{2} u}{\partial x^{2}}$ & $\delta^{2} \frac{1}{\delta^{2}}=1$ \\
$\frac{\mu_{B}}{\rho}\left(1+\frac{1}{A}\right) \frac{\partial^{2} u}{\partial y^{2}}$ & $\frac{\rho}{\rho} \frac{1}{1}=1$ \\
$\frac{\rho_{p}}{\rho} \frac{1}{\tau_{v}} u_{p}$ & $\frac{\rho}{\rho} \frac{1}{1} 1=1$ \\
$\frac{\rho_{p}}{\rho} \frac{1}{\tau_{v}} u$ & $\frac{1}{\delta^{2}} \delta^{2} 1=1$
\end{tabular}

Table 4. Order of magnitude for $y$ momentum equation of dusty Casson fluid

\begin{tabular}{cc}
\hline Term & $1 \frac{\partial}{\partial x}=\delta$ \\
$v \frac{\partial v}{\partial y}$ & $\delta \frac{1}{\delta}=\delta$ \\
$\frac{\mu_{B}}{\rho}\left(1+\frac{1}{A}\right) \frac{\partial^{2} v}{\partial x^{2}}$ & $\delta^{2} \frac{\delta}{1^{2}}=\delta^{3}$ \\
$\frac{\mu_{B}}{\rho}\left(1+\frac{1}{A}\right) \frac{\partial^{2} v}{\partial y^{2}}$ & $\delta^{2} \frac{\delta}{\delta^{2}}=\delta$ \\
$\frac{\rho_{p}}{\rho} \frac{1}{\tau_{v}} v_{p}$ & $\frac{\rho}{\rho} \frac{1}{1} \delta=\delta$ \\
$\frac{\rho_{p}}{\rho} \frac{1}{\tau_{v}} v$ & $\frac{\rho}{\rho} \frac{1}{1} \delta=\delta$ \\
$\frac{\sigma}{\rho} B_{0}^{2} \sin ^{2} \alpha_{1} v$ & $\frac{1}{\delta^{2}} \delta^{2} \delta=\delta$
\end{tabular}

Table 5. Order of magnitude for dusty Williamson fluid

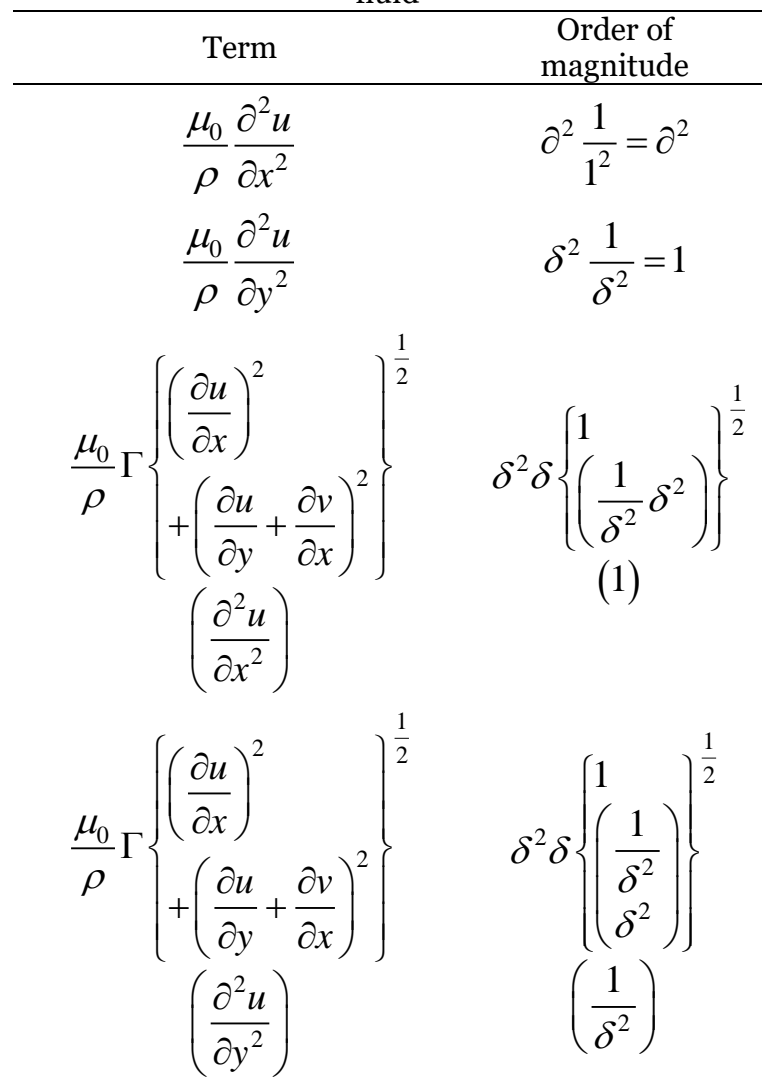




$$
\begin{aligned}
& \frac{\mu_{0}}{\rho} \Gamma \frac{\partial}{\partial x}\left\{\begin{array}{l}
\left(\frac{\partial u}{\partial x}\right)^{2} \\
+\left(\frac{\partial u}{\partial y}+\frac{\partial v}{\partial x}\right)^{2}
\end{array}\right\}^{\frac{1}{2}} \quad \delta^{2} \delta\left\{\left(\frac{1}{\left.\left(\frac{1}{\delta^{2}} \delta^{2}\right)\right\}^{\frac{1}{2}}}\right.\right. \\
& \left(\frac{\partial u}{\partial x}\right)
\end{aligned}
$$

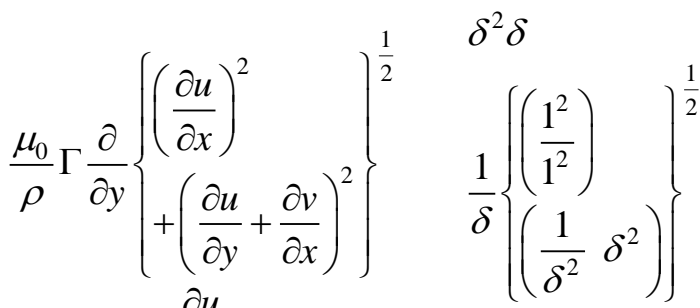

$$
\begin{aligned}
& \frac{\partial u}{\partial y} \quad \frac{1}{\delta}
\end{aligned}
$$

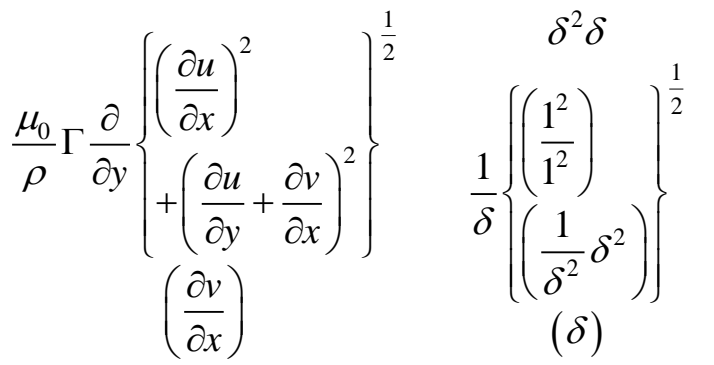

$$
\begin{array}{ll}
\frac{v}{1+\lambda_{2}} \lambda_{1}\left(\frac{\partial u}{\partial x} \frac{\partial^{2} u}{\partial y^{2}}\right) & \delta^{2} 1\left(\frac{1}{1} \frac{1}{\delta^{2}}\right)=1 \\
\frac{v}{1+\lambda_{2}} \lambda_{1}\left(\frac{\partial v}{\partial x} \frac{\partial^{2} u}{\partial x \partial y}\right) & \delta^{2} 1\left(\frac{\delta}{1} \frac{1}{\delta}\right)=\delta^{2}
\end{array}
$$

In the similar manner as dusty Casson fluid, the terms with order 1 from Tables 5 and 6are remained. Therefore, according to Tables 3-6, the momentum equation for fluid phase of dusty Casson, dusty Williamson and dusty Jeffrey fluids are given by

$$
\begin{aligned}
& u \frac{\partial u}{\partial x}+v \frac{\partial u}{\partial y}=v\left(1+\frac{1}{A}\right) \frac{\partial^{2} u}{\partial x^{2}}+\frac{\rho_{p}}{\rho \tau_{v}}\left(u_{p}-u\right) \\
& -\frac{\sigma}{\rho} B_{0}{ }^{2} \sin ^{2} \alpha_{1} u+\beta g\left(T-T_{\infty}\right), \\
& u \frac{\partial u}{\partial x}+v \frac{\partial u}{\partial y}=v \frac{\partial^{2} u}{\partial y^{2}}+\sqrt{2} v \Gamma \frac{\partial u}{\partial y} \frac{\partial^{2} u}{\partial y^{2}} \\
& +\frac{\rho_{p}}{\rho \tau_{v}}\left(u_{p}-u\right)-\frac{\sigma}{\rho} B_{0}{ }^{2} \sin ^{2} \alpha_{1} u+\beta g\left(T-T_{\infty}\right),
\end{aligned}
$$

Table 6. Order of magnitude for dusty Jeffrey fluid

\begin{tabular}{cc}
\hline$\frac{v}{1+\lambda_{2}} \frac{\partial^{2} u}{\partial x^{2}}$ & $\delta^{2} \frac{1}{1}=\delta^{2}$ \\
$\frac{v}{1+\lambda_{2}} \frac{\partial^{2} u}{\partial y^{2}}$ & $\delta^{2} \frac{1}{\delta^{2}}=1$ \\
$\frac{v}{1+\lambda_{2}} \lambda_{1}\left(u \frac{\partial^{3} u}{\partial x^{3}}\right)$ & $\delta^{2} 1\left(1 \frac{1}{1^{3}}\right)=\delta^{2}$ \\
$\frac{v}{1+\lambda_{2}} \lambda_{1}\left(u \frac{\partial^{3} u}{\partial x \partial y^{2}}\right)$ & $\delta^{2} 1\left(1 \frac{1}{\delta^{2}}\right)=1$ \\
$\frac{v}{1+\lambda_{2}} \lambda_{1}\left(v \frac{\partial^{3} u}{\partial x^{2} \partial y}\right)$ & $\delta^{2} 1\left(\delta \frac{1}{\delta}\right)=\delta^{2}$ \\
$\frac{v}{1+\lambda_{2}} \lambda_{1}\left(v \frac{\partial^{3} u}{\partial y^{3}}\right)$ & $\delta^{2} 1\left(\delta \frac{1}{\delta^{3}}\right)=1$ \\
$\frac{v}{1+\lambda_{2}} \lambda_{1}\left(\frac{\partial u}{\partial y} \frac{\partial^{2} u}{\partial x \partial y}\right)$ & $\delta^{2} 1\left(\frac{1}{\delta} \frac{1}{\delta}\right)=1$ \\
$\frac{v}{1+\lambda_{2}} \lambda_{1}\left(\frac{\partial u}{\partial y} \frac{\partial^{2} v}{\partial x^{2}}\right)$ & $\left.\delta_{1}\right)$ \\
$\frac{v}{1+\lambda_{2}}\left(\frac{\partial u}{\partial x} \frac{\partial^{2} u}{\partial x^{2}}\right)$ & $\left.\frac{1}{1^{2}}\right)=\delta^{2}$
\end{tabular}

In above equations, the Casson parameter is equated to $A=\mu_{B} \sqrt{2 \pi} / \rho_{y}$ and the fluid viscosity $v$ can be defined according to each fluid model as $v=\mu_{B} / \rho, v=\mu / \rho v=\mu_{0} / \rho$. If $A \rightarrow \infty, \Gamma=0$ and $\lambda_{1}=\lambda_{2}=0$, then the fluid case of Newtonian fluid can be recovered. It is therefore important to note that the momentum equations (12)-(14) are under the Boussinesq approximation, that being applied to the case of flow with buoyancy force term. The same approaches have been discussed by dissimilar authors (Jaluria, 1980; Bejan and Kraus, 
2003).

For dust phase, the momentum equation arises from inserting Equations (8) into (6) and it went on to use the boundary layer approximation that is treated similarly as in fluid phase by employing Equation (11). Tables 7 and 8 provide the analysis of order of magnitude for steady two dimensional of dust phase.

Table 7. Order of magnitude for $x$ momentum equation of

\begin{tabular}{cc} 
& dust phase \\
\hline$u_{p} \frac{\partial u_{p}}{\partial x}$ & Order of magnitude \\
\hline$v_{p} \frac{\partial u_{p}}{\partial y}$ & $1 \frac{1}{1}=1$ \\
$\frac{\rho_{p}}{\rho_{p}} \frac{1}{\tau_{v}} u_{p}$ & $11=1$ \\
$\frac{\rho_{p}}{\rho_{p}} \frac{1}{\tau_{v}} u$ & $11=1$ \\
\hline
\end{tabular}

Table 8. Order of magnitude for $y$ momentum equation

\begin{tabular}{cc} 
& of dust phase \\
\hline$u_{p} \frac{\partial v_{p}}{\partial x}$ & Order of magnitude \\
\hline$v_{p} \frac{\partial v_{p}}{\partial y}$ & $\delta \frac{\delta}{\delta}=\delta$ \\
$\frac{\rho_{p}}{\rho_{p}} \frac{1}{\tau_{v}} v_{p}$ & $1 \delta=\delta$ \\
$\frac{\rho_{p}}{\rho_{p}} \frac{1}{\tau_{v}} v$ & $1 \delta=\delta$
\end{tabular}

From the above tables, all terms in dust momentum equation remains unchanged since they are equally important. Despite that, the simplified form of this basic equation is expressed by dropping for $y$ direction, hence $u_{p} \frac{\partial u_{p}}{\partial x}+v_{p} \frac{\partial u_{p}}{\partial y}=-\frac{1}{\tau_{v}}\left(u_{p}-u\right) .(15)$

\section{Energy Equation}

The energy equation for fluid and dust phases are obtained by inserting Equations (9) into (7) and then the process of magnitude analysis is performed using
Equation (10) as provides in Tables9 and 10.

Table 9. Order of magnitude for energy equation of

\begin{tabular}{cc} 
& fluid phase \\
\hline Term & Order of magnitude \\
\hline$v \frac{\partial T}{\partial x}$ & $\delta \frac{1}{\delta}=1$ \\
$\frac{\partial T}{\partial y}$ & $\delta^{2} \frac{1}{1^{2}}=\delta^{2}$ \\
$\alpha \frac{\partial^{2} T}{\partial x^{2}}$ & $\delta^{2} \frac{1}{\delta^{2}}=1$ \\
$\alpha \frac{\partial^{2} T}{\partial y^{2}}$ & $11=1$ \\
$\frac{\rho_{p} c_{s}}{\rho c_{s}} \frac{1}{\tau_{T}} T_{p}$ & $11=1$ \\
$\frac{\rho_{p} c_{s}}{\rho c_{s}} \frac{1}{\tau_{T}} T$ & \\
\hline
\end{tabular}

Table 10. Order of magnitude for energy equation of \begin{tabular}{cc} 
& dust phase \\
\hline$u \frac{\partial T_{p}}{\partial x}$ & Order of magnitude \\
\hline$v \frac{\partial T_{p}}{\partial y}$ & $1 \frac{1}{1}=1$ \\
$\frac{\rho_{p} c_{s}}{\rho c_{s}} \frac{1}{\tau_{T}} T_{p}$ & $\delta \frac{1}{\delta}=1$ \\
$\frac{\rho_{p} c_{s}}{\rho c_{s}} \frac{1}{\tau_{T}} T$ & $11=1$ \\
\hline
\end{tabular}

According to Table 9, the term $\partial^{2} T / \partial x^{2}$ is discarded from the fluid energy equation since it is estimated to be small quantity. Therefore, the energy equation for both phases can be written as follows that consist of the terms with order 1 , we have $u \frac{\partial T}{\partial x}+v \frac{\partial T}{\partial y}=\alpha \frac{\partial^{2} T}{\partial y^{2}}+\frac{\rho_{p} c_{s}}{\tau_{T} \rho c_{p}}\left(T_{p}-T\right)$,

$u_{p} \frac{\partial T_{p}}{\partial x}+v_{p} \frac{\partial T_{p}}{\partial y}=-\frac{1}{\tau_{T}}\left(T_{p}-T\right) .(17)$ 


\section{CONCLUSION}

The indicating Equations (12)-(17) are now can be reduce into ordinary differential equations, which is less complex by using the appropriate similarity transformation which enable the numerical result to be computed.

\section{ACKNOWLEDGEMENT}

The authors gratefully acknowledge the financial support received from Universiti Malaysia Pahang through RDU 182307 and FRGS-RACER through RDU192602

\section{REFERENCES}

Anderson, J.D. (2009). Governing equations of fluid dynamics, in Computational fluid dynamics. Springer.

Arifin, N.S., Zokri, S.M., Kasim, A.R.M., Salleh, M.Z., Mohammad, N.F., \& Yusoff, W.N.S.W. (2017) Aligned magnetic field on dusty Casson fluid over a stretching sheet with Newtonian heating Malaysian Journal of Fundamental and Applied Sciences, 13, 244-247.

Arifin, N.S., Zokri, S.M., Kasim, A.R.M., Salleh, M.Z., \& Mohammad, N.F. (2018). Aligned magnetic field flow of Williamson fluid over a stretching sheet with convective boundary condition. in MATEC Web of Conferences. 2018. EDP Sciences.

Aurangzaib, K.A., Mohammad, N., \& Shafie, S. (2013). Unsteady MHD mixed convection flow with heat and mass transfer over a vertical plate in a micropolar fluid-saturated porous medium. J Appl Sci Eng, 16, 141-150.

Bejan, A. \& Kraus, A.D. (2003). Heat transfer handbook.John Wiley \& Sons.

Butt, A.S., Ali, A., and Mehmood, A. Hydromagnetic stagnation point flow and heat transfer of particle suspended fluid towards a radially stretching sheet with heat generation.(2017). in Proceedings of the National Academy of Sciences, India Section A:
Physical Sciences. Springer.

Hayat, T., Awais, M., Asghar, S., \& Hendi, A.A. (2011). Analytic solution for the magnetohydrodynamic rotating flow of Jeffrey fluid in a channel. Journal of Fluids Engineering, 133, 061201-1-7.

Hussanan, A., Salleh, M.Z., Khan, I., \& Shafie, S. (2018). Analytical solution for suction and injection flow of a viscoplastic Casson fluid past a stretching surface in the presence of viscous dissipation. Neural Computing and Applications, 29, $1507-1515$.

Isa, S.M., Ali, A., \& Shafie, S. (2016). Stagnation point flow of mhd dusty fluid toward stretching sheet with convective surface. Jurnal Teknologi, 78, 95-100.

Jaluria, Y. (1980). Natural convection: heat and mass transfer. Pergamon.

Kannan, K. (2003). Study of some two phase fluid flow problems without and with heat conduction. Ph.D thesis, Alaggapa University, India.

Kasim, A.R.M. \& Shafie, S. (2010). Mixed Convection Boundary Layer of a Viscoelastic Fluid past a Circular Cylinder with Constant Heat Flux. in Proceedings of the 1st Regional Conference on Applied and Engineering Mathematics. 
Kasim, A.R.M., Mohammad, N.F., \& Aurangzaib, Shafie.S. (2012). Natural convection boundary layer flow of a viscoelastic fluid on solid sphere with Newtonian heating. World Academy of Science, Engineering and Technology, 64, 628-633.

Kasim, A.R.M. (2014). Convective boundary layer flow of viscoelastic fluid. Ph.D thesis, Universiti Teknologi Malaysia.

Khan, M., Sardar, H., Gulzar, M.M., \& Alshomrani, A.S. (2018). On multiple solutions of nonNewtonian Carreau fluid flow over an inclined shrinking sheet. Results in Physics, 8, 926-932.

Marble, F.E. (1963). Dynamics of a gas containing small solid particles. 175-123.

Nadeem, S., Hussain, S., \& Lee, C. (2013). Flow of a Williamson fluid over a stretching sheet. Brazilian journal of chemical engineering, 30, 619-625.

Nakayama, Y. (2018). Introduction to fluid mechanics. Butterworth-Heinemann.

Nguyen, Q.-H. \&Nguyen, N.-D. Incompressible nonNewtonian fluid flows, in Continuum Mechanics-
Progress in fundamentals and Engineering applications. 2012. InTech.

Partal, P. \& Franco, J.M. (2010). Non-newtonian fluids. Rheology: encyclopaedia of life support systems (EOLSS), UNESCO. Eolss, Oxford 96-119.

Prandtl, L. (1904). On fluid motions with very small friction, Verhldg, 3, 484-491.

Saffman, P. (1962). On the stability of laminar flow of a dusty gas. Journal of Fluid Mechanics, 13, 120128.

Schlichting, H. (1974). Boundary-layer theory. Springer.

Siddiqa, S., Hossain, M.A., \& Saha, S.C. (2015). Twophase natural convection flow of a dusty fluid. International Journal of Numerical Methods for Heat \& Fluid Flow, 25, 1542-1556.

Sulochana, C., Prakash, J., \& Sandeep, N. (2016). Unsteady MHD flow of a dusty nanofluid past a vertical stretching surface with non-uniform heat source/sink. International Journal of Science and Engineering, 10, 1-9. 